http://jmscr.igmpublication.org/home/ ISSN (e)-2347-176x ISSN (p) 2455-0450

crossref DOI: https://dx.doi.org/10.18535/jmscr/v9i12.41

Journal Of Medical Science And Clinical Research

\title{
Incidence of Retinopathy of Prematurity in Preterms on Vitamin A Supplementation in NICU of Tertiary Care Centre
}

\author{
Authors \\ Dr Shruti Dhale ${ }^{1}$, Dr Nisha Yadav ${ }^{2 *}$, Dr Fareena Ansari ${ }^{3}$, Dr Poonam Mane ${ }^{4}$ \\ ${ }^{1}$ M.D. Pediatric, Associate Professor, Department of Pediatrics, Grant Government Medical College, \\ Mumbai \\ ${ }^{2 *}$ Senior Resident M.D. Pediatrics, Department of Pediatrics, Grant Government Medical College, Mumbai \\ ${ }^{3,4}$ Junior Resident M.D. Pediatrics, Department of Pediatrics, Grant Government Medical College, Mumbai \\ Corresponding Author \\ Dr Nisha Yadav
}

\begin{abstract}
Background: Retinopathy of prematurity $(R O P)$ is a multi-factoral vaso-proliferative retinal condition that affects mostly premature infants. In India, the prevalence of ROP in premature babies ranges from 35 to $60 \%$. Some of the risk factors associated with ROP includes low gestational age, late onset of sepsis, low birth weight, blood transfusion and use of surfactants and intraventricular haemorrhage. Preventive measures are still lacking behind. Some of the clinical trials showed reduced oxygen dependency in preterm babies who were given vitamin $A$ as preventive measures of $R O P^{2}$. Vascular endothelial growth factor (VEGF) demonstrated to have a role in pathogenesis of ROP, and Vitamin $A$ may help prevent neovascularisation due to oxygen induced retinopathy, by down regulating VEGF expression thus deterring ROP onset.

Objective: To study the incidence of Retinopathy in preterms receiving Vitamin A.

Material \& Methods: Institutional cross sectional study of 37 preterms with gestational age <34 weeks and birth weight $<1750 \mathrm{~g}$ were selected from January 2021 to November 2021. Consent of the mother or the father of the study participants were taken prior to including the infants in the study. All infants were started on oral Vitamin A supplement (5000 IU/oral on alternate days $x 3$ weeks), along with enteral nutrition once hemodynamically stable. The retinologist examines the infants after 3-4 weeks of birth or at 30-32 weeks of postconceptional age. First examination details were used to validate the findings, also the infants who had severe ROP; underwent laser therapy. Data was analysed by using the SPSS software and the incidence was then calculated.

Results: 11 out of 37preterms on vitamin A supplementation developed some degree of ROP in one or both eyes $(29.7 \%)$ ). Severity increased with decreasing gestational age. Based on the criteria of The International Classification for Retinopathy of Prematurity (ICROP), the disease reached stage 1,2 and 3 in 2.7\%(1), $8.1 \%$ (3) and $16.2 \%(6)$, respectively. No stage 4 and 5 ROP were detected.

Conclusion: The overall incidence of ROP in preterm who received Vitamin A supplementation was less by $29.7 \%$ when compared to the studies done earlier.-This shows that there is high likelihood of infants benefitting from Vitamin A supplementation in reducing Retinopathy of Prematurity.
\end{abstract}

\section{Introduction}

ROP is a multifactorial vaso-proliferative retinal disorder that increases in incidence with decreasing gestational age. The condition is, by and large, limited to preterm babies with a birth weight of less than 1750 gram or gestational age 
of less than 34 weeks. As the gestational age decreases, the incidence of ROP, a multifactorial vaso-proliferative retinal disorder increases. This is more often seen in preterm babies who weigh less than 1750 gram or whose gestational age is less than 34 weeks ${ }^{1}$.

There is an approximate 25 million live births which happens in India out of which $8.5 \%$ of the births results in baby weighing less than $2 \mathrm{~kg}$ in weight. Incidence of ROP in the new born babies with weight less than $1750 \mathrm{~g}$ lies between 35 to $60 \%$. On the other hand, in $80 \%$ of the babies weighing less than $1000 \mathrm{~g}$ are more vulnerable and are prone to develop $\mathrm{ROP}^{1}$. Some of the risk factors which are associated with ROP include low gestational age, low birth weight, late onset sepsis, intra-ventricular haemorrhage, blood transfusion and the use of surfactants ${ }^{2}$.

Vitamin A accounts to be one of the most important micro-nutrient which is needed for an orderly growth and differentiation in the infants. It is a fat-soluble vitamin and plays a vital role in regulating the growth of the epithelial cells as well as helps in the development of retina and lungs. It also plays a vital role in maintaining the normal vision $^{3,4}$.

The important role of vitamin A in the prevention of ROP is that it has the potential to reduce oxygen dependency in premature babies ${ }^{5}$. Vascular endothelial growth factor (VEGF) demonstrated to have a role in pathogenesis of ROP. Vitamin A may reduce neovascularisation caused by oxygen induced toxicity, by down regulating VEGF expression and so delaying ROP onset $^{5,6}$.

Plasma Vitamin A levels in preterm infants rise as the gestational age progresses ${ }^{7}$. As a result of limited transplacental transfer from their mothers, low intake through enteral feeding for several weeks after birth, and poor gastrointestinal absorption, puts the preterm babies at the risk for developing Vitamin A deficiency. According to a study, at least $65 \%$ of the infants with birth weight less than $1250 \mathrm{~g}$ and $80 \%$ of the infants with a birth weight of less than $1000 \mathrm{~g}$ are at risk of developing some degree of $\mathrm{ROP}^{7,8}$.

According to the International Classification of ROP (ICROP) 2005 guideline, ROP is classified in five stages in increasing stages of severity; the higher the stage of ROP, the more severe the disease is. ${ }^{9}$

Currently, there are no preventive measures adopted to tackle the given condition. Some of the studies suggests preventive measures, include erythropoietin, propranolol, caffeine, antioxidants and omega 3 poly unsaturated fatty acids; Vitamin A has been suggested to have a preventive effect on $\mathrm{ROP}^{10}$.

Some clinical trials have demonstrated the efficacy of vitamin $A$ in the prevention of complications of prematurity, particularly ROP and $\mathrm{BPD}^{11}$. In this article, we are studying the incidence of ROP in pre-terms receiving vitamin A supplementation.

\section{Materials and Methods}

This was a prospective observational study, conducted in the paediatric department of a tertiary care hospital. Neonates admitted in NICU were enrolled for the study as per the criteria given. It was a prospective, stratified randomized cohort study performed from January 2021 to November 2021. 37 preterm patients were selected. The Institutional Ethics Committee (IEC) approved the study. Written informed consent was obtained from both parents. Pre-terms with gestational age $<34$ weeks and low birth weight babies <1750gram who were admitted in our NICU were selected for the study.

\section{Inclusion Criteria}

1) Preterms admitted <34 weeks gestation age.

2) Preterms $<72$ hours of age

3) Receiving mechanical ventilation, noninvasive respiratory support or supplemental oxygen( fio $2>21 \%$ ) at 24 hours of age.

4) Receiving oral Vitamin A supplementation (5000 IU on alternate days) .Started along 
with enteral feeds as soon as infant is hemodynamically stable.

\section{Exclusion Criteria}

1) Congenital Major anomalies

2) Congenital TORCH infections with overt signs at birth

3) Genetic metabolic diseases.

4) Terminal illness as evidenced by $\mathrm{pH}<7.0$ for $>2$ hours or persistent bradycardia associated with hypoxia for $>2$ hours

5) Grade III or IV Intracranial hemorrhage
All infants born $>28$ weeks or > 1200g were screened at 3-4 weeks of chronological age. Preterms $<28$ weeks of gestation or $<1200$ g were screened at 2-3 weeks. The retinologist was unaware about the vitamin A supplementation. The following examination was repeated based on the findings of the first examination. Infants with high grade ROP underwent laser therapy. Data was tabulated and analyzed using SPSS 20.0 version software.

\section{Results}

Figure 1: Gender Distribution of the studies cases

\section{GENDER}

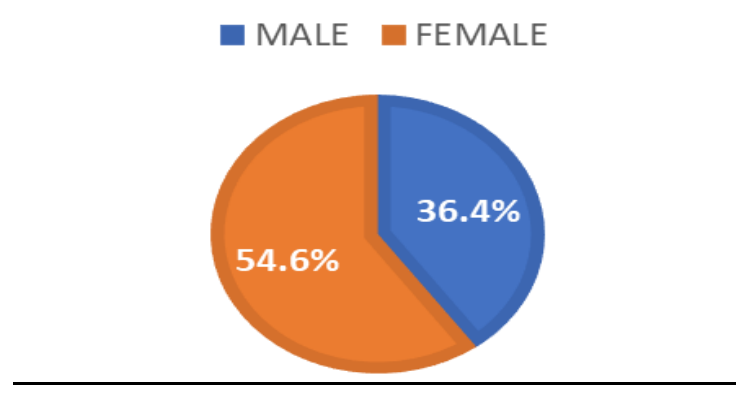

As per data collected, of 37 preterm on vitamin A were male $(36.4 \%)$ who were screened for ROP. supplementation, 11 infants were diagnosed to Male to female ratio $0.8: 1$. have ROP. Of which 6 were female $(54.6 \%)$ and 5

Figure 2: Relation between ROP and Gestational Age

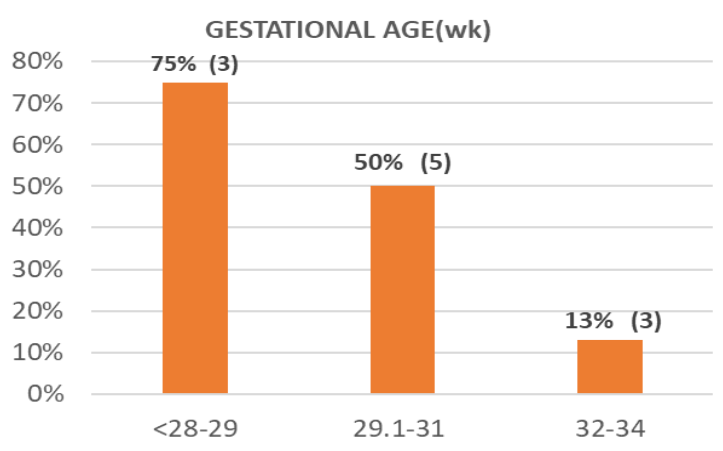

Out of 37 preterms, 3 out 4 patients with gestational age $<29$ weeks developed ROP (75\%). 5 out of 10 babies (50\%) between 29.1 to 31 weeks, and 3 out of 23 preterms $(13 \%)$ between
32 -34 gestational age had developed ROP. Gestational age $<29$ weeks was significantly associated with higher rates of ROP. 
Fig 3: Birth weight and Retinopathy of prematurity

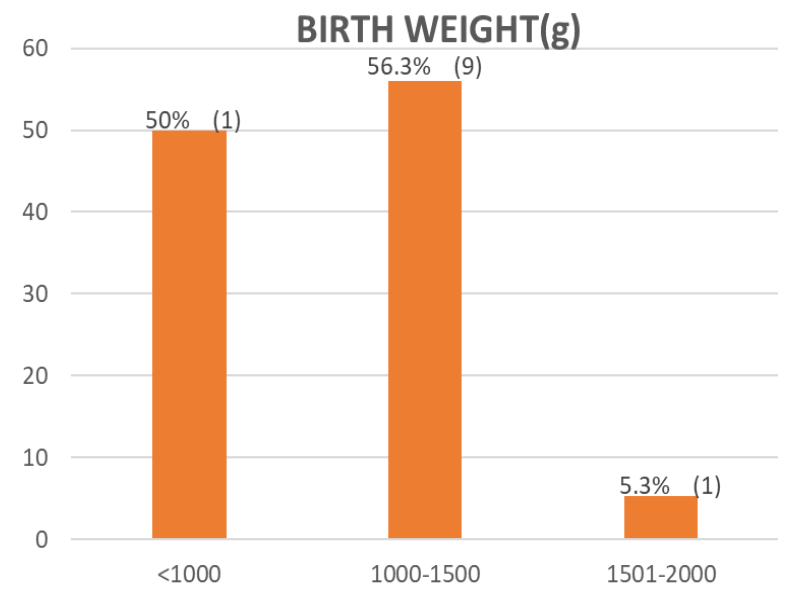

In this study, $1(50 \%, 1$ out of 2$)$ infants less than $1000 \mathrm{~g}$ had ROP. 9 pre-terms $(56.3 \%, 9 / 16)$ between $1000-1500 \mathrm{~g} \mathrm{n}$ and 1 preterm $(5.3 \%, 1 / 19)$ with birth weight $>1501 \mathrm{~g}$ developed ROP. The smallest infants with birth weight less than $1000 \mathrm{~g}$ are at the highest risk.

Fig 4: Severity of ROP with Progressing Gestational age

\begin{tabular}{|l|l|l|c|c|c|c|}
\hline Gestational age & $\begin{array}{c}\text { Total } \\
\text { Patients }\end{array}$ & $\begin{array}{c}\text { ROP } \\
\text { Patients }\end{array}$ & Stage 1 & Stage 2 & $\begin{array}{c}\text { Stage } \\
\mathbf{3}\end{array}$ & $\begin{array}{c}\text { Plus } \\
\text { Disease }\end{array}$ \\
\hline$<28-29$ weeks & 4 & $3(75 \%)$ & & & 2 & 1 \\
\hline 29.1 to 31 weeks & 10 & $5(50 \%)$ & & 1 & 4 & \\
\hline $32-34$ weeks & 23 & $3(13 \%)$ & 1 & 2 & & 1 \\
\hline Total & 37 & $29.7 \%$ & 1 & 3 & 6 & \\
\hline
\end{tabular}

A total of 37infants fulfilled the screening criteria. ROP was detected in $29.7 \%$ of neonates. 1 preterm $(2.7 \%)$ had stage 1 disease, 3 infants $(8.1 \%)$ with stage 2,6 neonates $(16.2 \%)$ with stage 3 ROP. Prethreshold disease was present in $1(2.7 \%)$ preterm of gestational age 30.3 with birth weight of $1.49 \mathrm{~g}$. There were no cases with stage 4 and 5 ROP

\section{Discussion}

The present study was a hospital based prospective study conducted in the department of paediatrics of a tertiary care hospital. The total number of cases of preterm infants in the present study is 37 over the study period of 10 months. Out of the 37 premature patients, there were 11 infants diagnosed with varying stages of Retinopathy of Prematurity. Of which, there were
6 females and 5 male babies with male: female ratio of $0.8: 1$. Incidence was higher in infants with lower gestational age with $75 \%$ of preterms below 29 weeks showing ROP followed by $50 \%$ preterms between gestational age 29.1 to 31 weeks and $13 \%$ between $32-34$ weeks showing ROP. Higher incidence may be attributed due to preterms having low body stores of Vitamin A as they have low levels of both retinol and retinol binding protein (RBP). Certain studies have shown association between low plasma vitamin A concentrations and development of $\mathrm{ROP}^{12}$.

In our study, association between birth weight and ROP was also evaluated. Incidence was $50 \%$ in infants below $1000 \mathrm{~g}, 56.3 \%$ in preterms weighing between $1000-1500 \mathrm{~g}$ and $5.3 \%$ in neonates between 1501 to $1750 \mathrm{~g}$. However, since gestational age is a major contributor of birth 
weight, it was not possible to evaluate the independent effect of birth weight on ROP in the given study.

The study also shows increasing severity of ROP with decreasing Gestational age. Treatment was performed in all preterms with severe $\mathrm{ROP}(>$ stage 3) with regular follow up. The treatment involved laser photocoagulation.

The results of previous studies regarding incidence of ROP have varied. The overall incidence in India of ROP varies between 35 and $60 \%$ in babies weighing less than $1750 \mathrm{~g}^{1}$. Almost $80 \%$ of infants weighing less than $1000 \mathrm{~g}$ are reported to develop $\mathrm{ROP}^{1}$. This study was aimed to evaluate the incidence ROP in preterms on oral vitamin A supplementation. Certain studies have shown that parenteral/I.M administration of vitamin $\mathrm{A}$ is able to prevent complications of prematurity ${ }^{13}$ Oral formulation are well tolerated and no adverse events have been observed ${ }^{5}$. Certain studies have shown that parenteral administration of Vitamin A supplementation is able to prevent complications of prematurity ${ }^{13}$.

Overall incidence of ROP in preterms receiving Vitamin A supplementation was comparatively less i.e $29.7 \%$ as compared to results in the previously done studies.

\section{Conclusion}

In conclusion vitamin A supplementation is beneficial in reducing ROP in premature infants. As they have reduced body stores of Vitamin A and are at risk for developing vitamin $\mathrm{A}$ deficiency, therefore they should receive Vitamin A parenteral supplementation as early as possible within first few days of life.It may help in reducing the severity of ROP in extreme as well as incidence of Retinopathy of Prematurity.

\section{Reference}

1. Singh M. Care of the new born revised 8ed (2017). CBS Publishers \& Distributors Private Limited; 2017 Nov 30.

2. Ugurbas SC, Gulcan H, Canan H, Ankarali $\mathrm{H}$, Torer B, Akova YA. Comparison of
UK and US screening criteria for detection of retinopathy of prematurity in a developing nation. Journal of American Association for Pediatric Ophthalmology and Strabismus. 2010 Dec 1;14(6):506-10.

3. Spears K, Cheney C, Zerzan J. Low plasma retinol concentrations increase the risk of developing bronchopulmonary dysplasia and long-term respiratory disability in very-low-birth-weight infants. The American journal of clinical nutrition. 2004 Dec 1;80(6):1589-94.

4. Mactier H, Weaver LT. Vitamin A and preterm infants: what we know, what we don't know, and what we need to know. Archives of Disease in Childhood-Fetal and Neonatal Edition. 2005 Mar 1;90(2):F103-8.

5. Garofoli F, Mazzucchelli I, Decembrino L, Bartoli A, Angelini M, Broglia M, Tinelli C, Banderali G, Stronati M. Levels and effectiveness of oral retinol supplementation in VLBW preterm infants. International journal of immunopathology and pharmacology. 2018 Dec;32:2058738418820484

6. Sun H, Cheng R, Wang Z. Early vitamin a supplementation improves the outcome of retinopathy of prematurity in extremely preterm infants. Retina (Philadelphia, Pa.). 2020 Jun;40(6):1176

7. Tyson, J E et al. "Vitamin A supplementation for extremely-low-birthweight infants. National Institute of Child Health and Human Development Neonatal Research Network." The New England journal of medicine vol. 340,25 (1999): 1962-8.

doi:10.1056/NEJM199906243402505

8. Kiatchoosakun $\mathrm{P}$, Jirapradittha J, Panthongviriyakul MC, Khampitak T, Yongvanit P, Boonsiri P. Vitamin A supplementation for prevention of bronchopulmonary dysplasia in very-lowbirth-weight premature Thai infants: a 
randomized trial. J Med Assoc Thai. 2014

Oct 1;97(Suppl 10):S82-8.

9. International Committee for the Classification of Retinopathy of Prematurity. The international classification of retinopathy of prematurity revisited. Archives of ophthalmology (Chicago, Ill.: 1960). 2005 Jul;123(7):9919.

10. Aranda JV, Qu J, Valencia GB, Beharry KD. Pharmacologic interventions for the prevention and treatment of retinopathy of prematurity. InSeminars in perinatology 2019 Oct 1 (Vol. 43, No. 6, pp. 360-366). WB Saunders.

11. Darlow BA, Graham PJ, Rojas- Reyes MX. Vitamin A supplementation to prevent mortality and short- and long- term morbidity in very low birth weight infants. Cochrane database of systematic reviews. 2016(8).

12. Shenai JP, Kennedy KA, Chytil F, etal. Clinical trial of vitamin A supplementation in infants susceptible to bronchopulmonary dysplasia

13. Yang SN. Nutritional approach to the prevention of complications of prematurity with emphasis on vitamin A supplementation. Pediatrics \& Neonatology. 2014 Oct 1;55(5):331-2. 